\title{
Complex karyotype in acute promyelocytic leukemia
}

\begin{abstract}
Acute Promyelocytic leukemia (APL) is characterized by $\mathrm{t}(15 ; 17)$ translocation. A subset of APL patients may have additional chromosomal abnormalities, the clinical significance of which remains controversial. Here we present a case of APL with complex karyotype including $\mathrm{t}(1 ; 9)(\mathrm{q} 12 ; \mathrm{q} 12)$ for the first time who died during treatment because of coagulopathy and we emphasize the need of large multicenter collaborative trials to establish prognostic significance of additional cytogenetic abnormalities (ACA) in APL patients both generally and specifically.
\end{abstract}

Keywords: chromosomal abnormalities, complex karyotype, peripheral blood, bone marrow, azurophilic granulation, coagulopathy
Volume 4 Issue 3 - 2017

\author{
Shawana Kamran,' Kamran Nazir, ${ }^{2}$ Imran \\ Nazir I' \\ 'Shifa International Hospital, Pakistan \\ ${ }^{2}$ Pakistan Air Force Hospital, Pakistan
}

Correspondence: Shawana Kamran, Shifa International Hospital, Islamabad, Pakistan, Email drshawana33@gmail.com

Received: February 17, 2017 | Published: April 19, 2017
Abbreviations: APL, acute promyelocytic leukemia; ATRA, all-trans retinoic acid; ACA, additional cytogenetic abnormalities; MPO. myeloperoxidase; PETHEMA, programa de estudio y tratamiento de las hemopatías malignas; RT-PCR, reverse transcriptase polymerase chain reaction

\section{Introduction}

Acute Promyelocytic leukemia (APL) is characterized by typical morphological features, $t(15 ; 17)$ translocation and active response to all-trans retinoic acid (ATRA) in the great majority of patients. However, a subset of APL cases may present with atypical phenotypic, cytogenetic or molecular features at different stages of the disease. The biological and clinical significance of these features sometimes remains obscure. ${ }^{1}$ Amongst APL patients with atypical cytogenetic features, in addition to $t(15 ; 17)$, other chromosome abnormalities are also found either at the time of diagnosis or at relapse, with the incidence ranging from $29 \%$ to $43 \%$ in previous reports. Trisomy 8 was the most frequent secondary change reported in many studies. ${ }^{2}$ Although $\mathrm{t}(15 ; 17)$ is considered to be a favorable cytogenetic feature, however, the prognostic significance of additional cytogenetic abnormalities (ACA) in APL has remained a matter of debate.

\section{Materials and methods}

\section{G-banding karyotype analysis}

Karyotypic analysis was performed on un-stimulated short-term peripheral blood and bone marrow cultures ( 24 and 48hours), followed by Giemsa trypsin banding. Cytogenetic findings were described according to the International System for Human Cytogenetic Nomenclature (ISCN2009).

\section{Reverse transcriptase polymerase chain reaction (RT- PCR) for PML-RAR $\alpha$}

Peripheral blood was used for RNA extraction using RT-PCR protocols for PML-RAR $\alpha$ amplification.

\section{Case presentation}

A 46years old male presented with 3 days history of epistaxis and fever. On examination, there was no hepatosplenomegaly or lymphadenopathy. His complete blood counts revealed WBC:54,900/

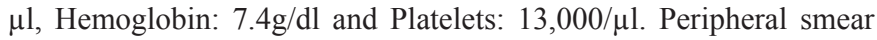

showed markedly increased abnormal promyelocytes with intense azurophilic granulation and few cells containing numerous auer rods (faggot cells). Bone marrow aspirates and trephine biopsy also revealed significantly increased abnormal hypergranular promyelocytes. Flow cytometric analysis showed that these abnormal promyelocytes were positive for CD13, CD33, CD117, strongly positive for Myeloperoxidase (MPO), had low expression of CD34 and were negative for HLA-DR which was consistent with APL. RT-PCR for PML-RARA was positive for BCR3 locus. Cytogenetic studies showed complex chromosomal abnormalities i.e. 47,XY,der(1) $\mathrm{t}(1 ; 9)(\mathrm{q} 12 ; \mathrm{q} 12), \quad$ add3q?,del5q23,+8,der(9), del11p13, $\mathrm{t}(15 ; 17)$ (q24;q21), del18q21[17]/46,XY,idem,-16[2]. Coagulation profile was deranged. Fibrinogen was $65 \mathrm{mg} / \mathrm{dl}$ (Normal range: 200-400 mg/dl). His treatment was started with All-Trans retinoic acid (ATRA) but unfortunately the patient died of uncontrolled bleeding complications.

\section{Results}

\section{G-banding karyotype analysis}

Karyotypic analysis was performed which showed $\mathrm{t}(15 ; 17)$ (q24; 21$)$. Additional abnormalities included $\operatorname{der}(1) \mathrm{t}(1 ; 9)(\mathrm{q} 12 ; \mathrm{q} 12)$, add3q,del5q23,+8,der(9), del11p13, del18q21 in all metaphases. Only two metaphases showed monosomy16 along with those already mentioned (Figure1). RT-PCR for PML-RAR $\alpha$ and RT-PCR performed on peripheral blood showed PML-RAR $\alpha$ was positive for BCR-3.

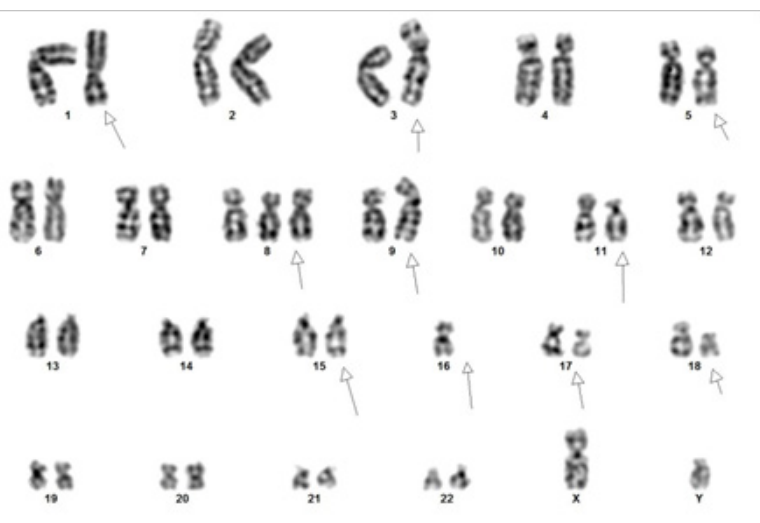

Figure I Karyotypic analysis showed $t(\mid 5 ; 17)(q 24 ; q 21)$ along with additional abnormalities. 


\section{Discussion}

APL is characterized by the presence of abnormal promyelocytes resulting from the maturation block caused by $\mathrm{t}(15 ; 17)(\mathrm{q} 24 ; \mathrm{q} 21)$. The incidence of ACA in APL has been consistently reported to be in the range of $26 \%-39 \%$ with trisomy 8 being the most frequent abnormality $(33 \%-53 \%)$ among the secondary chromosomal changes. ${ }^{2}$ Our case report is the first of its kind in our country to be reported as APL presenting with bleeding complications and ACA. According to a Spanish study the characteristics, outcome and prognostic value of cytogenetics in a large cohort of successfully karyotyped patients with a long follow-up was carried out by the Programa de Estudio $y$ Tratamiento de las Hemopatias Malignas (PETHEMA) group (studies LPA96 and LPA99). ${ }^{3}$ According to this study, with regards to clinicopathological characteristics, there was significant association of ACA with low platelet counts, the presence of coagulopathy and higher relapse risk scores which were not previously reported but patients with and without additional chromosomal abnormalities had similar complete remission rates $(90 \%$ and $91 \%$, respectively). Moreover, previous studies have also shown the association between the breakpoint in BCR3 locus and ACA in APL. ${ }^{2}$ According to literature, It is believed that ACA in general, or some specific abnormality in particular (e.g., trisomy 8) along with BCR3 isoforms, might have some role in generating the factors leading to a poorer risk score however the Spanish study identified neither ACA overall nor any specific abnormality as an independent risk factor for relapse.

Few early studies have indicated the ACA to be important prognostic factor for outcome of APL patients presenting with structural chromosomal abnormalities other than $t(15 ; 17)$ as the ACA render leukemic cells insensitive to drugs leading to poor response to therapy, including resistance to ATRA, Arsenic trioxide or chemotherapy. ${ }^{1,4}$ However, three large studies by the Japan leukemia study group APL97, the APL93 trial and the UK MRC group including another study on newly diagnosed APL with ACA cases treated with arsenic oxide as a front line therapy, found no significant influence of ACA in APL patients. ${ }^{5-8}$ One of the reasons for this discrepancy could be the intensity of the induction/ consolidation/ maintenance chemotherapy. According to literature, only one case report other than our case, showed APL with del5q as an ACA. In this study, the concomitant appearance of PML-RARA with some of the recurring chromosomal abnormalities that characterize MDS is rare. Moreover, ACA in APL could account for the biological malignant grade and prognostic index. As per the authors of this study, most of growth factors like GM-CSF alleles and cytokines like IL-3, IL-4 and IL-5 are located on chromosome $5 \mathrm{q}$ and they proposed that APL with del5q might have good prognosis compared to APL without del5q which should be determined by clinical observation. ${ }^{9}$ Another case report by Wafa et al. ${ }^{10}$ showed $\mathrm{t}(1 ; 2)(\mathrm{q} 42 \sim \mathrm{q} 43 ; \mathrm{q} 11.2 \sim 12)$ in an APL patient who died earlier in the course of treatment. This is similar to our patient who also died early in the course of treatment and showed $\mathrm{t}(1 ; 9)(\mathrm{q} 12 ; \mathrm{q} 12)$ as an ACA. ${ }^{11}$ The involvement of chromosome 1 in both cases may signify a bad prognostic feature which needs to be elucidated by further clinical observations and molecular studies. ${ }^{10}$

\section{Conclusion}

To our knowledge this is the first case report showing $t(1 ; 9)$ (q12;q12) along with other chromosomal abnormalities. We conclude that additional cases will have to be identified and followed up with multicentre collaboration to ascertain the prognostic significance of additional/complex chromosomal abnormalities in APL patients to stratify these cases for appropriate treatment.

\section{Acknowledgements}

None.

\section{Conflict of interest}

The author declares no conflict of interest.

\section{References}

1. Xu L, Zhao WL, Xiong SM, et al. Molecular cytogenetic characterization and clinical relevance of additional, complex and/or variant chromosome abnormalities in acute promyelocytic leukemia. Leukemia. 2001;15(9):1359-1368.

2. Slack JL, Arthur DC, Lawrence D, et al. Secondary cytogenetic changes in acute promyelocytic leukemia-prognostic importance in patients treated with chemotherapy alone and association with the intron 3 breakpoint of the PML gene: a cancer and leukemia group B study. $J$ Clin Oncol. 1997;15(5):1786-1795.

3. Cervera J, Montesinos P, Rivas JMH, et al. Additional chromosome abnormalities in patients with acute promyelocytic leukemia treated with all-trans retinoic acid and chemotherapy. Haematologica. 2010;95(3):424-431.

4. Hiorns LR, Swansbury GJ, Mehta J, et al. Additional chromosome abnormalities confer worse prognosis in acute promyelocytic leukaemia. Br J Haematol. 1997;96(2):314-321.

5. Ono T, Takeshita A, Iwanaga M, Asou N, et al. Impact of additional chromosomal abnormalities in patients with acute promyelocytic leukemia: 10-year results of the Japan adult leukemia study group APL97 study. Haematologica. 2011;96(1):174-176.

6. De Botton S, Chevret S, Sanz M, et al. Additional chromosomal abnormalities in patients with acute promyelocytic leukaemia do not confer poor prognosis: results of APL 93 trial. Br J Haematol. 2000;111(3):801-806.

7. Burnett AK, Grimwade D, Solomon E, et al. Presenting white blood cell count and kinetics of molecular remission predict prognosis in acute promyelocytic leukemia treated with all-trans retinoic acid: result of the randomized MRC trial. Blood. 1999;93(12):4131-4143.

8. Lou Y, Suo S, Tong H, et al. Characteristics and prognosis analysis of additional chromosome abnormalities in newly diagnosed acute promyelocytic leukemia treated with arsenic trioxide as the front-line therapy. Leuk Res. 2013;37(11):1451-1456.

9. Imataki O, Uemura M. Chromosomal abnormality of acute promyelocytic leukemia other than PML-RARA: A case report of acute promyelocytic leukemia with del(5q). BMC Clin Pathol. 2016;16:16.

10. Wafa A, Moassass F, Liehr T, et al. Acute Promyelocytic Leukemia with translocation $\mathrm{t}(15 ; 17)(\mathrm{q} 22 ; \mathrm{q} 21)$ associated with $\mathrm{t}(1 ; 2)$ (q42 q43;q11.2 12): a case report. Journal of Medical Case Reports. 2016;10(1):203. 